\title{
Channelopathies go above and beyond the channels
}

Since they were first described in the 1990s studies of rare, monogenetic channelopathies have been presented as opportunities for understanding more common polygenic disorders. Slightly more than two decades after the first descriptions of channelopathies, we use this special issue to re-assess: What have we learned from studies of channelopathies? What have channelopathies taught us about common diseases?

The assessment begins with a survey of the genetics of channelopathies - not just an extensive summary of what is known about mutations in ion channel genes but of potential modifiers and accessory subunits (NEUROPHARM-D-17-00087). Because the identities of many ion channel interacting proteins are known, channelopathies have played an important role in revealing how mutations in related proteins can modify disease severity. Indeed many rare, monogenetic channelopaties show distinct polygenic influences in how they manifest.

At a closer level, new structural data allows for exact modelling of how individual mutations change the behaviour of the affected channels (NEUROPHARM-D-17-00098). The nature of ion channel function means that decades of biophysics has described the function of many channels in detail, and these studies are now complemented by molecular dynamics, which can show precisely how the changes in channels lead to the changes in function linked to pathology - in at a level of detail which geneticists working with proteins of unknown function can only envy.

An additional confounding factor in mapping genotype-phenotype relationships that has emerged relatively recently, is that even in some cases when a mutation in an ion channel appears pathological, the functional consequences for the channels affected remain elusive. It has become apparent that in many cases, post translational processing may be the culprit (NEUROPHARM-D-1700127), with mutations that can produce apparently normal channels leading to dysfunctions through changes in trafficking (for example). This not only reveals a new mechanism for disease, but may reveal new potential angles to treat the changes - by targeting the post translational consequences rather than the channel gating itself.

Intimately related to trafficking, is the assembly of complete channels, including subunits that do not contribute directly to the pore, but which are necessary for channel function. In this issue we highlight the sodium channel $\beta$ subunits as proteins which can lead to channelopathies (NEUROPHARM-D-17-00230), including epilepsy, through mechanisms such as trafficking and anchoring, even though they are not directly forming the pore of the channel. The study of channelopathies has thus shone a bright light on how mutations in proteins that in heterologous systems may not be necessary for protein function, may become functionally critical in the endogenous cells. The fact that mutations in $\beta$ subunits can produce phenocopies of mutations in the pore-forming $\alpha$ subunits is a clear demonstration of the requirement for $\beta$ subunits to produce functional channels.

Not all alterations of function are pathological. Ion channels are hugely diverse, and many are restricted to different tissues (NEUROPHARM-D-17-00184). Mining the Human Protein Atlas reveals the level of tissue specific specialisation of ion channels - with important implications for treatments 
(i.e. an ion channel restricted to cardiac muscle is a poor target for an anti-epileptic treatment). But the restrictions are more specific than one gene limited to one tissue. Voltage gated calcium channels are used to demonstrate a level of tissue specific expression that is honed down to different splice variants dominating in different regions. This opens the potential for splice variant specific treatments for tissue specific manifestations of channelopathies.

It is not even the case that all diseases caused by dysfunction of ion channels can be traced to mutations in ion channel genes. An important and relatively new family of channelopathies is caused by antibodies to ion channels and their associated proteins (NEUROPHARM-D-17-00059). Binding of antibodies can disrupt channel function, and trigger immune responses. In these cases, even where channel function is known to be disrupted, treatments are clearly targeted at the antibodies, including plasma exchange and treatment with steroids. Knowing the antibody based origin of these channelopathies makes direct targeting of the dysfunctional channel illogical.

Studies of ion channels have historically relied heavily on the remarkable array of toxins that target them (NEUROPHARM-D-17-00311). Toxins have served to identify, purify, functionally characterise, and even to name different ion channels. Now new efforts are developing to harness animal toxins to treat ion channels that have gone wrong. The high affinity and specificity of toxins - evolved to modify channels in order to seriously disrupt excitable cells - is now being used to bind to and in some cases restore the function of channels in excitable cells. The approach holds promise so large it has not yet been quantified, as the number of toxins and their specificities is remains unknown.

A great advantage of channelopathies is that the function of the disrupted channels can be interrogated in real time. Historically this has been done in ex vivo tissue or dissociated cells, where mutant and wild type channels can be compared in relative isolation, however recently studies of peripheral nerve excitability carried out in patients with mutations in neuronal ion channels, has opened a possibility to compare results from reduced preparations to functional consequences in functioning neurons in patients (NEUROPHARM-D-17-00086). These studies have the advantage of not just corroborating in vitro findings, but also of revealing how neurons adapt to compensate for a change in the function of a single type of channel, for example by up- or down-regulating other channels. Knowing the compensatory strategies present in vivo might modify mechanistic approaches to treatment.

In the final article in this issue, we look forward to new approaches not just to 'treating' channelopathies, but which raise the possibility of curing them (NEUROPHARM-D-17-00164). Preclinical studies of gene therapy have suddenly emerged from a decade of damage control with vigorous, and in many cases impressively effective treatments of monogenetic diseases. The detailed biophysical understanding behind channelopathies, including types of functional changes, potential compensatory mechanisms, consequences of channel changes on neuronal excitability, means that channelopathies offer rich grounds for rational delivery of genes to offset the effects of mutations. In addition gene-editing allows the potential to simply repair the mutation. Hurdles in systemic delivery, and targeted expression remain, but the extensive data on the functional mechanisms, modifications, and successful therapies for channelopathies, means that these are some of the most transparent targets for gene therapy and repair. 
To return to the original question: what have we learned about neurological disorders from studies of channelopathies? They have provided worked examples of how finding a mutation in a gene is only the beginning to understanding the mechanism of a disease, and have revealed the many possible places - from associated proteins to tissue-specific splicing - to look when a mutation does not explain the full clinical manifestations of a disease. 